\title{
RECHERCHES RÉCENTES SUR LES AQUEDUCS ROMAINS DE GAULE MÉDITERRANÉENNE
}

\author{
Guilhem FABRE, Jean-Luc FICHES et Philippe LEVEAU
}

Ce dossier n'a pas la prétention de jeter les bases d'un inventaire des aqueducs de Gaule Narbonnaise. Son objectif est de présenter quelques travaux originaux qui s'inscrivent dans la dynamique d'une recherche dont l'élément moteur a été le programme conduit autour de l'aqueduc de Nîmes à partir de 1983 (Fabre et al. dir., 2000) et, en même temps, de montrer la diversité des voies ouvertes. Ces études de cas prennent leur sens par rapport à un espace que définit la Eombinaison de deux données physiques majeures, le climat te contexte géologique. Dans une partie de la Narbonnaise, en effet, la sécheresse estivale, qui constitue la caractéristique principale de la zone climatique nord-méditerranéenne, Terturbe le ravitaillement en eau de l'habitat et impose à Magriculture des contraintes que l'irrigation peut pallier fig. 1). Cet espace est également caractérisé par la place -11. occupent les formations calcaires qui favorisent la disparition des eaux de surface et la constitution d'importants quifères karstiques. Des nappes profondes, alimentant de qombreuses sources pérennes, atténuent ainsi les effets de la sécheresse estivale.

L'un des apports traditionnellement imputés au crédit de Rome est d'avoir assuré, par la construction d'aqueducs, l'indépendance de l'habitat et, à un moindre degré, celle de l'agriculture par rapport aux contraintes du milieu. L'étude de ces ouvrages permet donc à la fois d'évaluer les progrès techniques accomplis dans le domaine hydrologique et de mesurer leur diffusion dans une région de l'Occident romain. Traditionnellement, ce type d'ouvrage est considéré comme urbain ; le dossier s'ouvre d'ailleurs par deux études monographiques (Foucras et Garczynski pour Antibes, p. 13-34; Bermond pour Balaruc-les-Bains, p. 35-48), mais cette partie est également illustrée par des études plus spécialisées, qui montrent la diversité des champs de recherche (Paillet pour le Pont du Gard, p. 49-68; Carbon et al. pour Nîmes, p. 69-86; Guendon pour Arles, p. 87-96). Nous avons tenu cependant à élargir cette présentation aux adductions rurales, dont la meunerie de Barbegal constitue un témoin exceptionnel (Leveau et Thernot, p. 97-105) au regard des vestiges, plus modestes mais toujours dignes d'intérêt, que l'on rencontre plus fréquemment depuis les marges de la zone méditerranéenne (Passelac, p. 107-118) à la plaine languedocienne (Harfouche et al., p. 119-130; Mauné et al., p. 131-145) et au cœur de la Provence (Mocci et al., p. 147-160).

\section{L'ARCHÉOLOGIE DES AQUEDUCS \\ DES PRÉOCCUPATIONS ANCIENNES, UN DÉVELOPPEMENT RÉCENT}

Les aqueducs occupent une place tout à fait particulière parmi les monuments caractéristiques de l'époque romaine. Les grands ouvrages d'art dont ils ont occasionné la construction sont ruraux et non urbains pour des raisons évidentes de captage souvent lointain. À ce titre, ils constituent, avec les routes, l'une des principales manifestations du contrôle exercé par la ville romaine sur la campagne. Par ailleurs, si le traité de Vitruve constitue une référence incontournable pour l'architecture romaine en général, les aqueducs sont les seuls monuments qui aient bénéficié, dans l'Antiquité, d'une étude spécifique, celle de Frontin. De ce fait, l'archéologie des aqueducs a été relativement longue à se mettre en place: Frontin en parlait si bien ! Une analyse archéologique pourraitelle faire mieux qu'en illustrer fidèlement les propos, même si ceux-ci ne concernaient que Rome? Bien que, dès le XV'HII et surtout au XIX" s., des ingénieurs et des architectes se soient intéressés aux ouvrages d'art qui en sont la partie spectaculaire, les monographies qui prennent en compte l'ensemble d'un aqueduc et se préoccupent de son fonctionnement et de son histoire sont encore bien peu nombreuses.

L'une des raisons de l'intérêt que la société a porté aux aqueducs est l'admiration que ces ouvrages romains ont suscitée auprès des ingénieurs qui, au XIX ${ }^{\circ}$ s., entreprirent de doter des villes d'adductions d'eau: obligés d'assurer aux agglomérations qui se développaient une alimentation en eau pour satisfaire des préoccupations qui n'étaient pas fondamentalement différentes de celles des Romains - la salubritas et l'amoenitas vantées par Pline -, ils lancèrent des enquêtes sur les travaux hydrauliques anciens. Les aqueducs les mieux connus sont ceux dont subsistaient des vestiges importants, que l'on pouvait ambitionner de restaurer afin de réutiliser le canal.

Sur ces ouvrages connus, l'acteur technique principal est le topographe : il dispose des outils et des savoirs pour reconstituer et étudier un canal qui conduit l'eau par gravité. Ce n'est pas par hasard si l'une des premières monographies consacrées à un aqueduc fut celle de $\mathrm{K}$. Grewe sur celui de Cologne où le parcours a été reconstitué à partir de prospections et d'opérations de nivellement (Grewe, 1986). L'étude de l'aqueduc de 


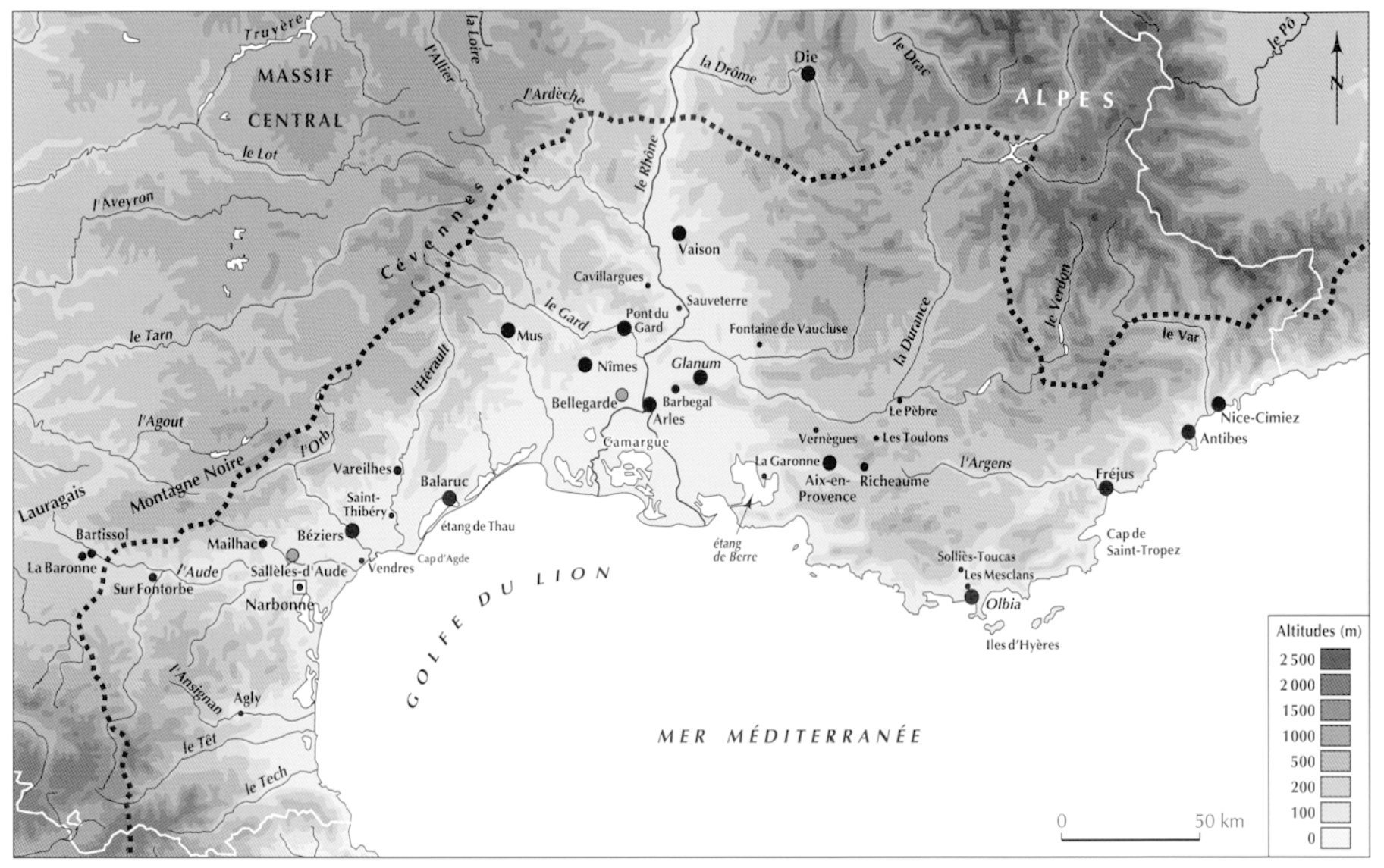

aqueduc urbain

aqueduc de destination incertaine
- aqueduc rural présenté dans le dossier - autre aqueduc cité dans le dossier

\#.. limite bioclimatique del'aire méditerranéenne

Fig. 1 - La France méditerranéenne. Situation des aqueducs urbains et ruraux présentés ou cités dans le dossier (DAO T. Panouillères, CNRS).

Calesarea de Maurétanie avait connu, cependant, une genèse différente: la publication monographique n'était pas aux origines de cette recherche (Leveau, Paillet, 1976). Le but initial était d'appréhender l'équipement rural d'une ville méditerranéenne, dans lequel les ressources en eau occupaient naturellement une place majeure. Les prospections devaient permettre de distinguer de nombreuses installations hydrauliques en relation avec des villae et les sections du grand aqueduc qui alimentait la ville.

\section{GRANDE ET PETITE HYDRAULIQUE}

Les contributions de ce dossier s'articulent autour de deux thèmes archéologiques principaux qui distinguent les aqueducs alimentant des villes et ceux qui alimentaient des établissements situés à la campagne. On pourrait croire que cela permet de différencier " grands " et " petits " aqueducs. Mais la réalité n'est pas si simple: tous les chefs-lieux de cité ne disposaient pas, comme Arles et Nîmes, d'un aqueduc de grande dimension ; celui d'Antibes est bien plus modeste et les petits aqueducs alimentaient aussi bien des agglomérations secondaires comme Balaruc-les-Bains que des villae. On ne peut donc juger de la destination d'un aqueduc à la seule indication de sa taille. Ainsi, Aqua Sextice était alimentéc par quatre aqueducs, dont l'un seulement, celui de La Traconnade, était de grand gabarit ; parmi les trois autres, deux, qui cernent la montagne de la Sainte-Victoire, ont une section à peine supérieure à celle des aqueducs de villae présentés dans ce dossier (Guyon et al., 1998, p. 245-248; Nin, 2000).

Cela nous incite à reprendre une proposition déjà formulée (Leveau, 2000) pour comparer les aqueducs et évaluer l'effort financier qu'ils impliquaient en s'appuyant sur le dossier des adductions de Rome, bien documenté sur ce point. Ces ouvrages ont une canalisation (specus) dont la section interne varie entre $1,50 \mathrm{~m}^{2}$ pour l'Aqua ('laudia et $3,35 \mathrm{~m}^{2}$ pour l'Anio Novus, soit en termes de linéaire de maçonnerie nécessaire $3,48 \mathrm{~m}$ et $5,20 \mathrm{~m}$ (chiffre obtenu en additionnant les deux hauteurs des pićdroits et la largeur du fond). Dans une perspective purement hydraulique, on pourrait concevoir un classement des aqueducs en fonction de la section de leur cuvelage : plus de $1 \mathrm{~m}^{2}$, grande hydraulique; moins de $1 \mathrm{~m}^{2}$, petite hydraulique. Mais il faut tenir compte aussi d'autres éléments comme la longueur et la présence ou non d'ourages d'art. $\mathrm{Si}$, appliquant ces règles, on tente de dresser une liste des ouvrages de grande hydraulique, on s'aperçoit que celle-ci est assez brève. En ('aule Narbonnaise, on n'en compte que cinq ou six : ceux de Nîmes $\left(1,38 \mathrm{~m}^{2}\right)$ el d'Arles $\left(1,08 \mathrm{~m}^{2}\right)$, le grand aqueduc de Vienne $\left(3,16 \mathrm{~m}^{2}\right)$, celui de Fréjus $\left(1 \mathrm{~m}^{2}\right.$ au 
plus) et l'un des aqueducs d'Aix-en-Provence. Pour les Trois Gaules et la Germanie, pas plus de quatre ou cinq, à Lyon (Brévenne, voire Gier), Autun, Metz (presque $1 \mathrm{~m}^{2}$ : Lefebvre, 1996) et Cologne. Cette liste exclut, on le voit, les aqueducs secondaires de Vienne et d'Aix-en-Provence, mais aussi des ouvrages comme ceux de Glanum $\left(0,52 \mathrm{~m}^{2}\right.$ : Agusta-Boularot, Paillet, 1997) et de Béziers $\left(0,45 \mathrm{~m}^{2}\right.$ : Andrieu, 1990 et 1999 , p. 95) ainsi que des canalisations dont la destination est incertaine: Sallèles-d'Aude (aqueduc de Narbonne?) et Bellegarde (aqueduc de Beaucaire?) ${ }^{1}$. Les aqueducs d'Aquitaine s'inscrivent dans cette catégorie, comme ceux de Saintes (Bailhache, 1983, p. 41) ou de Cahors (Rigal, 1999) et, sous réserve d'examen plus approfondi, les aqueducs de Fleury à Poitiers (Leveau, 1992, p. 277) et de Bourges (Adam, 1984, p. 269-270).

\section{AQUEDUCS DES VILLES}

De nombreuses villes de Gaule Narbonnaise attendent les monographies que méritent leurs aqueducs. C'est encore le cas, par exemple, des villes du Vaucluse, malgré l'étude récente dont vient de faire l'objet l'aqueduc du Groseau qui alimentait Vaison-la-Romaine (Dupuy, Chardon, 2003). Les articles qui sont ici consacrés aux adductions urbaines - trois chefs-lieux de cités (Antibes, Arles et Nîmes), une agglomération secondaire (Balaruc-les-Bains) - illustrent autant d'aspects de l'archéologie de ces ouvrages. Ainsi, J. Foucras et P. Garczynski livrent une monographie sur un aqueduc qui avait fait l'objet d'une brève notice dans le Manuel d'archéologie de A. Grenier; I. Bermond montre que des agglomérations dites secondaires comme Balaruc-les-Bains pouvaient disposer d'une adduction importante, peut-être dans ce cas à la faveur d'un acte d'évergésie ; J.-L. Paillet, de son côté, présente des réflexions sur la construction du Pont du Gard ${ }^{2}$ et tente de reconstituer la démarche de l'architecte et les étapes du chantier, alors qu'au vallon des Arcs l'étude architecturale est intervenue après une fouille qui a mis en évidence les étapes d'un changement d'usage occasionné par la construction des moulins de Barbegal.

Les recherches récentes ont montré que ces aqueducs, utilisés pendant plusieurs siècles, avaient connu des aléas dans leur fonctionnement. Le tracé a même pu être modifié et cela pour deux raisons principales, dont, en Narbonnaise, l'aqueduc de Fréjus donne une bonne illustration (Gébara et al., 2002) : la nécessité de renforcer un débit insuffisant (entraînant à Fréjus le captage d'une source plus lointaine) et les difficultés rencontrées dans l'entretien d'une canalisation implantée dans un milieu instable (déplacement d'ouvrages de franchissement). Mais nulle part on ne connaît encore de

1. 0,45 $\mathrm{m}^{2}$ pour ce dernier (Fabre, Sauvage, 1999, p. 238). À Sallèlesd'Aude même, la section du curelage est de $0,45 \mathrm{~m}^{2}$ au plus (Fabre, observations de terrain inédites) alors qu à (abezac, en amont, elle est de $0,70 \mathrm{~m}^{2}$ (Andrieu, 1999, p. 94). L'aqueduc qui passe à Sallèlesd'Aude pose l'irritante question de sa destination finale, non résolue pour l'instant : la villa des Jasses (Courrent in Dellong et coll., 2002, p. 583-586) ou Narbonne (Andrieu, Cazal, 1999).

2. À compléter par les observations de J.-C. Bessac (2003) sur les traces laissées sur place par le chantier. remaniements de l'ampleur de ceux qui furent réalisés sur l'aqueduc de Cherchell (Leveau, Paillet, 1976, p. 64-67 et p. 76-77) et vraisemblablement à Lyon à la boucle de la Durèze (Burdy, 1996, p. 75-91).

L'histoire complexe de ces ouvrages ressort aussi de la confrontation des résultats des analyses architecturales, de l'étude stratigraphique des dépôts carbonatés et des sondages archéologiques qui ont permis, sur l'aqueduc de Nîmes (Fiches et al., 2000), de dater le chantier de construction et la période d'abandon (milieu $\mathrm{I}^{\mathrm{er}}$ s.-début $\mathrm{VI}^{\mathrm{e}}$ s. apr. J.-C.), mais aussi de distinguer des travaux de réglage peu après la mise en eau, une période de fonctionnement optimal puis un manque d'entretien et des dégradations, enfin un essai de réhabilitation peu de temps avant l'abandon (Fiches, Paillet, 2000). Il n'est pas toujours facile, cependant, d'obtenir des éléments de datation précis pour chacune de ces phases, et souvent même pour fixer l'époque de la construction et de l'arrêt de l'utilisation, à l'exemple des aqueducs d'Arles et de Fréjus. Sur ce dernier, on a pu distinguer différentes phases dont la plus importante correspond au captage des sources de la Siagnole à Mons (Var) alors qu'à l'origine celui-ci ne concernait que la Foux à Montauroux (Var). Mais les sondages archéologiques, les études d'architecture et l'analyse des concrétions et des briques n'ont fourni qu'une série d'événements situés dans une chronologie relative plus ou moins précise. Pour caler cet ensemble de données, il a fallu se référer aux datations obtenues en ville pour les grandes étapes du développement urbain. Or, comme on l'a déjà souligné par ailleurs (Fabre, Fiches, 2003), tous les spécialistes ne sont pas d'accord aujourd'hui sur ces datations. Le cas de l'aqueduc d'Arles, où seul un remaniement est daté assez précisément de l'époque d'Hadrien, n'est pas différent; étudiant les enregistrements sédimentaires du bassin du vallon des Arcs, J.-L. Guendon en montre bien l'apport essentiel pour une histoire de l'aqueduc, mais il souligne aussi les difficultés que l'on rencontre pour passer à la datation de l'ouvrage initial.

\section{AQUEDUCS DE VLLAE ET D'ÉTABLISSEMENTS RURAUX}

La seconde partie de ce dossier attire l'attention sur la diffusion de l'innovation constituée par les aqueducs dans les campagnes, le plus souvent des conduits de petite taille, adaptés aux besoins évidemment moins importants que ceux d'une ville. La plupart des ouvrages présentés ici sont de ce type, mais le dossier en fournit un contre-exemple avec l'alimentation des moulins de Barbegal.

L'étude de ces aqueducs nous paraît constituer un enjeu majeur de la recherche en archéologie rurale. Pour l'Italie, R. J. A. Wilson déplorait que ces ouvrages aient peu attiré l'attention (Wilson, 1996). Ce n'est pas tout à fait exact, $X$. Lafon a traité de leur rôle dans l'alimentation en eau douce des villae maritimae (Lafon, 2001, p. 301-305). En Narbonnaise, il est prématuré d'évaluer leur importance. Là, pas plus que dans le reste des Gaules, à l'exception notable de la cité des Bituriges (Maussion in Batardy et al., 2001, p. 120-125), ces systèmes d'alimentation en eau n'ont fait l'objet d'inventaires systématiques. En Gaule du Sud cependant, de nombreuses enquêtes fournissent des données exploitables au moins à titre 
comparatif, dont la liste augmente avec la parution des volumes de la Carte archéologique de la Gaule. Elles montrent que ces adductions constituent un élément important de l'équipement hydraulique des campagnes, susceptibles d'alimenter des villae mais aussi des installations spécialisées ou des agglomérations plus ou moins grandes. Ainsi, l'aqueduc de Richeaume à Puyloubier, retrouvé en contrebas de la villa, a alimenté des bassins ultérieurement reconvertis pour une utilisation artisanale ou agricole, mais il se poursuivait au-delà et sa destination pouvait concerner des dépendances du bâtiment principal, une autre villa, un hameau, voire des réservoirs ou de petites retenues utilisés pour l'irrigation.

La question de la fonction de ces ouvrages introduit un plus large débat sur l'économie rurale. S'agit-il de canalisations alimentant un bâtiment résidentiel, ses thermes, ses bassins d'agrément? Leur construction répondait-elle à des besoins agricoles ou artisanaux? Les différentes contributions portant sur ce sujet nourrissent cette problématique. Les villae étaient des lieux de résidence de riches familles. Leurs propriétaires y souhaitaient retrouver le confort et le charme des domus urbaines à l'agrément desquelles concouraient les bassins et les jeux d'eaux. Richeaume en fournit un excellent exemple qui illustre également la transformation de ces espaces au cours du temps. En revanche, la villa de Vareilles à Paulhan met en évidence un autre type de découverte, celle de moulins hydrauliques, qui marque une avancée importante de l'archéologie rurale. Un passage souvent cité du traité de Palladius précise qu'il fallait utiliser l'eau des thermes pour faite tourner un moulin, mais cette affirmation s'est heurtée à une idée reçue : celle de l'invention médiévale du moulin hydraulique. Pour la Gaule, la nouvelle datation des moulins de Barbegal (II's.) a ouvert une brèche dans la théorie en démontrant le caractère erroné de la relation établie entre la fin de l'esclavage et le recours à l'énergie hydraulique (Leveau, 1995). La publication du moulin des Mesclans dans le Var (Brun, Borréani, 1998) a suivi les travaux de l'architecte F. Rakob (1974) dont A. Wilson (1995) avait publié un compte rendu qui faisait le point sur la question de la diffusion du moulin à roue horizontale. Ces travaux ont relancé l'attention sur ce type d'ouvrage en Gaule dont les découvertes sont devenues assez. nombreuses.

Il faut également penser à l'irrigation. Au vu des connaissances dont on dispose, on serait tenté d'opposer dans ce domaine l'économie rurale de la Gaule du Sud à celle de la péninsule Ibérique et plus largement des régions du Maghreb, où, comme en Italie (Thomas, Wilson, 1994), sont connus des ouvrages maçonnés destinés à l'arrosage de cultures (Leveau et al., 1993, p. 176-180 et p. 209-212) ${ }^{3}$. Les recherches actuelles incitent toutefois à nuancer cette opposition. L ces travaux géoarchéologiques conduits par J.-F. Berger et C. Jung leur ont permis d'identifier un réseau d'irrigation d'époque romaine dans la plaine d'Orange. Dans le remplissage de fossés, un matériel sableux allogène caractéristique indique que le captage se trouvait en amont. L'éloignement de celui-ci et la superficie de la zone concernée montrent qu'il relève d'une grande irrigation qui disparaît durant le Moyen Âge (Berger,

3. Voir aussi, pour les vallées du Guadalquivir et du Guadiana : Gorges, Rico, 1999 ; (Garvalho-Quintela et al., 1999.
2000, p. 75). Ce précieux témoignage est susceptible de relancer la recherche sur une irrigation pratiquée à proximité de centres urbains romains, comme on en connaît sur le pourtour de la Méditerranée. En tout cas, on ne doit pas oublier que l'aqueduc de Nîmes, durant le III's. au moins, a fait l'objet de prélèvements importants, au profit des campagnes (Fabre et al. dir., 2000, p. 412-413). En amont du Pont du Gard en effet, un plateau de garrigue a pu être partiellement irrigué à partir de ponts à arcades multiples comme en témoignent sept amas carbonatés, constitués autour d'échafaudages en bois et à l'origine desquels on a retrouvé, dans la paroi du canal, les traces d'une vanne qui suppose une utilisation concertée des prises d'eau.

\section{AQUEDUCS ET GÉOARCHÉOLOGIE : L'APPORT D'UNE RÉFLEXION SUR LE MILIEU PHYSIQUE}

Les archéologues et les spécialistes des géosciences se sont largement préoccupés du milieu physique dans lequel l'ouvrage avait été construit et, en premier lieu, des contraintes topographiques et géomorphologiques. Les résultats de leurs études confirment à la fois les recommandations de Frontin dans ce domaine et le savoir-faire des ingénieurs et architectes romains qui ont eu en charge la construction des aqueducs.

\section{CONTRÔLES TOPOGRAPHIQUES ET GÉOLOGIQUES}

Le tracé des aqueducs - dont l'orientation ( $\mathrm{Nm} \mathrm{ou} \mathrm{Ng}$ ) doit être donnéc dans le sens de l'écoulement - s'inscrit dans deux types d'espaces aux caractéristiques topo-morphologiques varićes. Le plus simple correspond à un milieu faiblement pentu et relativement homogène dans lequel le canal est bâti en tranchéc, le plus souvent dans des terrains détritiques comme c'est le cas des aqueducs ruraux de Mailhac, du Carcassonnais et de Richeaume, mais aussi de la partie de l'aqueduc de Nîmes située dans la Vistrenque. Un autre type, plus complexe, se rencontre dès que l'on a de longues conduites qui traversent des terroirs différents, négociant parfois même des changements de bassins versants. I.es modes d'implantation de l'aqueduc sont alors plus variés: ainsi, dans des roches cohérentes carbonatées, on peut trouver des tronçons plaqués et engravés en L. sur des interfluves plus ou moins marqués. ou engravés en U comme c'est le cas dans des formations détritiques.

La première nécessité était d'implanter le conduit dans unc topographie qui, souvent, ne se prêtait pas à une telle opération. Le progrès intervenu avec Rome se manifeste par la réalisation d'ouvrages d'art qui relèvent de deux types, le pont et le tunnel. Lc Pont du Gard est un bon exemple de la pertinence de l'analyse dı milieu par l'ingénieur romain. Le franchissement des zones déprimées sur un pont portant un canal à écoulement libre est d'ailleurs très majoritairement attesté en Narbonnaise. Surtout connue en Gaule par l'exemple classique des aqueducs de Lyon, la technique du " siphon inversé ", qui fonctionnait selon le principe des vases communicants, n'est guère attestée, dans le Midi, qu'à Arles pour le franchissement 
du Rhône et l'alimentation du quartier de Trinquetaille en rive droite (Cochet, 1993), mais d'autres exemples sont possibles, comme à Vaison-la-Romaine pour la traversée de l'Ouvèze (Dupuy, Chardon, 2003, p. 79). On a également utilisé des conduites en céramique noyées dans un béton de chaux. Il faudra attendre cependant l'époque moderne pour la mise au point des canalisations de fonte capables de résister à des pressions considérables.

La technologie des galeries souterraines était bien connue et maîtrisée par les ingénieurs romains (Grewe, 1998). Probablement mise au point par les mineurs, elle a été utilisée de manière systématique pour des travaux de drainage dont l'exemple le plus célèbre est la galerie du lac Fucin (Leveau, 1993) et pour le passage des aqueducs. Cette technologie coûteuse a été largement mise en œuvre en Italie (Riera, 1994). En Narbonnaise, elle a été relativement peu utilisée. Sur l'aqueduc de Nîmes, la longueur totale des tunnels est inférieure à $1 \mathrm{~km}$ (Fabre $e t$ al. dir., 2000, p. 97-99 et p. 113-114; Bessac, 2000, p. 383-387). La technique a probablement été mise en œuvre sur une grande échelle pour la construction du plus important des aqueducs alimentant Aix-en-Provence (Traconnade) : depuis le captage à Jouques, dans le bassin de la Durance, il devait passer sous le plateau de Venelles par une galerie souterraine de plusieurs kilomètres, partiellement explorée à partir de puits de plusieurs dizaines de mètres de profondeur.

Par ailleurs, la galerie des Taillades à Fontvieille est significative de la maîtrise d'une technologie qui n'était pas réservée aux aqueducs urbains (Grewe, 1998, p. 98-104; Gateau, Gazenbeek, 1999, p. 167-168) : creusée dans la molasse à partir de puits de surface sur quelques centaines de mètres de longueur, elle a servi à évacuer les eaux d'une dépression ; bien conservée, elle est toujours en usage. Enfin, sur l'aqueduc de Fréjus, le creusement de la tranchée ouverte dans le substrat à la Roche-Taillée exploite une fracture qui affecte le bord d'un escarpement (Gébara et al., 2002, p. 130-132).

\section{HYDRAULIQUE}

La circulation de l'eau dans un aqueduc répond aux lois générales de l'écoulement gravitaire à surface libre. En fait, le concepteur jouait au mieux avec les altitudes afin d'obtenir une pente de radier convenable pour l'écoulement optimum de l'eau et éviter des vitesses de flux voisines de $1,5 \mathrm{~m} / \mathrm{s}$, susceptibles d'éroder trop vite le monument. Cette question des pentes ${ }^{4}$, évoquée à plusieurs reprises dans ce dossier, pose en fait le problème fondamental, à la fois simple et complexe, du fonctionnement hydraulique d'un aqueduc. L'écoulement gravitaire à surface libre est toujours variable dans l'espace comme dans le temps. En effet, sur la tocalité de la longueur de l'ouvrage, la pente du radier et donc le profil en long du specus ne sont pas constants; ce n'est que sur de courts tronçons que le régime peut être uniforme et qu'on peut appliquer valable-

4. Discussion in Hodge, 1992, chap. 6, et surtout 7 et 8. Après avoir donné les valeurs minimales de la pente d'un radier d'aqueduc, bien différentes sclon Vitruic $(0,5 \%)$ ct Plinc $(0,02 \%)$, il propose, avec prudence, $0,3-0,15 \%$ (voir ibid., id., p. 216). ment, comme l'ont fait ici plusieurs auteurs, les formules classiques de calcul de débit, comme celles de Darcy, Chézy, Prony, Manning-Strickler ${ }^{5}$ ou Bazin ${ }^{6}$. Ailleurs, quand valeurs des pentes et profils diffèrent, le régime est graduellement varié, ce qui empêche leur utilisation en toute rigueur pour l'ensemble d'un aqueduc ${ }^{7}$. Il faut considérer aussi le fait que l'anatomie interne du conduit évolue entre la mise en eau et la fin du fonctionnement, notamment avec l'accrétion des dépôts carbonatés internes, et que, de plus en plus, les recherches mettent au jour des structures hydrauliques de régulation de l'écoulement sur la totalité du tracé, et non exclusivement au captage ou à l'arrivée (Arles, Nîmes...).

Lorsque la pente devenait trop forte, pour éviter la dégradation du canal, il fallait établir un dispositif de freinage des flux d'écoulement ou d'absorption d'énergie. Ces dispositifs sont restés longtemps mal connus. C'est en effet à une date très récente qu'un ingénieur s'est livré à une recherche spécifique (Chanson, 2000). Pour freiner l'eau, on établit une succession de courts plans inclinés, séparés par des seuils de rupture de pente de section carrée, rectangulaire ou circulaire. Lorsque la pente est plus faible, les plans inclinés sont remplacés par des paliers à déclivité normale séparant des seuils. Bien attestés sur les aqueducs lyonnais, ces dispositifs n'ont pas encore été observés en Narbonnaise. Mais le même effet est obtenu par un dispositif bien attesté : un bassin comportant un conduit de décharge et un double vannage, aval et latéral. Toutes ces structures traduisent le souci permanent d'une gestion hydraulique contrôlée, pour deux raisons principales: la nécessité de vidanger plus ou moins complètement le specus pour des opérations de vérification, d'entretien ou de réparation, et la possibilité de moduler les débits en fonction des besoins ou lors des crues survenues au niveau des captages. Leur présence et, en tout état de cause, l'existence d'un profil à pentes irrégulières, de règle sur les grands aqueducs avec seuils et ruptures de pentes, sont des éléments qui permettent de penser à une hydraulique mixte, combinant le stockage et un écoulement variable (Bossy $e t$ al., 2000 ; Chanson, 2000, 2002). Ainsi, l'écoulement dans un aqueduc est toujours complexe: non permanent (modifications des paramètres dans le temps), globalement non uniforme (modifications des paramètres d'une section à l'autre), graduel (modifications lentes des paramètres d'une section à l'autre) et rapide (modifications rapides des paramètres d'une section à l'autre dues à un seuil, ressaut, etc.).

5. Formule utilisée par les hydrologues du Leichtweiss Institut de Braunschweig pour le site du vallon des Arcs.

6. Formule « de base » de Bazin pour le calcul de la vitesse moyenne de l'eau (V) et donc du débit $(Q)$ :

$V=C \sqrt{R} . i(m / s)$, avec $C=87 \sqrt{R} / Y+\sqrt{R}$ donc $Q=V . S\left(m^{3} / s\right)$

$\mathrm{C}=$ facteur de $\mathrm{R} / \mathrm{Y}, \mathrm{R}=$ rayon hydraulique, $\mathrm{i}=$ pente du radier, $S=$ section mouillée, $Y=$ coefficient de rugosité.

Cette formule est quasiment identique à celle de Chéry; seuls les coefficients de rugosité varient chez Bazin et introduisent plus de finesse dans le calcul, d'où son succès. Malgré des formulations différentes, les équations anglo-saxonnes sont, en définitive, de conception équivalente.

7. Voir les mises au point dans I Iodge, 1992 (en anglais) ; Lencastre, 1984 ; Graf, Altinakar: 1993, 1996 (en français). 
Dans le prolongement de cette question en cours de développement, se pose celle des captages, car les textes antiques nous apprennent que les Anciens prêtaient une grande attention à la qualité de l'eau.

\section{LES CAPTAGES}

L'analyse documentaire des sites captés, rivières et sources, interroge et présente des constantes. L'interrogation est double. D'abord celle qui porte sur la construction du captage dont il ne reste souvent rien ou peu de choses ; aucune publication ne fait état de captage antique globalement bien conservé (bassin, drains, chambre de captage, etc.) ; souvent l'emplacement de griffons n'est même pas clairement établi. Force est de reconnaître aussi que beaucoup d'incertitudes planent également sur leur localisation exacte, comme, dans ce dossier, à La Baronne, Vareilles et Richeaume ou à l'exemple évoqué plus loin des aqueducs d'Arles. Pour l'aqueduc de Nîmes, bien qu'une tradition évoque les " sources d'Eure et d'Ayran " et suggère que des captages aient été pratiqués, dans l'Antiquité, en amont des sources d'Eure, on a considéré, en raison de débits jugés suffisants et en l'absence de témoins antiques à l'amont, que seule la zone d'exsurgence située à Uzès entre le château de Plantéry et le bassin moderne d'Eure avait été exploitée (Fabre et al. dir., 2000).

Si nous ne connaissons pratiquement rien des captages des aqueducs d'Arles et de Barbegal, c'est peut-être parce qu'ils n'ont pas été reconnus, mais aussi et surtout parce qu'ils ont fait l'objet de réutilisations et sont toujours en usage. Cette impossibilité de se prononcer sur l'ancienneté des captages a servi d'argument pour allonger vers l'amont la source captée pour l'aqueduc d'Arles au nord des Alpilles en lui attribuant des sources dont seule une utilisation moderne est attestée. Il en va de même de l'aqueduc sud. Son point de départ se trouve à la Burlande (le Paradou) où l'on a fouillé un bassin qui assurait la convergence de deux canaux. L'origine de ces canaux est probable: l'un viendrait de la fontaine d'Arcoule, l'autre du vallon d'Entreconque sur la commune de Mouriès où un conduit maçonné est connu. Mais il existe d'autres sources importantes qui pouvaient être captées par cet ouvrage. Les prospections conduites par M. Gazenbeek ont montré la fragilité des hypothèses que l’on pouvait élaborer en rattachant systématiquement les éléments connus de canalisation à l'aqueduc. Il peut tout aussi bien s'agir de conduits menant à des villae ou arrosant des champs et des jardins. C'est ainsi que M. Gazenbeek a été conduit à contester l'existence d'une branche affluente de l'aqueduc qui aurait capté une source dans le vallon d'Auge (Gateau, Gazenbeek, 1999 , p. $164, n^{\circ} 18^{*}$ ). Ce secteur, dont l'hydrogéologie a été complètement bouleversée par l'exploitation de la bauxite, paraît avoir été mis en valeur par une importante villa anciennement connuc.

Pour les prises d'eau bien identifiées en revanche, les constantes sont patentes. Dans la grande majorité des cas, les ingénicurs antiques ont, quand ils en avaient la possibilité, délaissé les cours d'eau au régime souvent capricieux, de type torrenticl, avec des charges de turbidités fortes, directement et rapidement influencées par les précipitations toujours irrégu- lières et le plus souvent brutales ${ }^{8}$. Ils ont fixé leur choix sur les sources pérennes, le plus souvent karstiques, suivant en cela les prescriptions hippocratiques ${ }^{9}$. Les raisons sont multiples, au-delà du fait qu'une source, élément ponctuel à morphologie relativement stable, est plus facile à capter qu'un cours d'eau dont les lits sont mobiles avec une évolution plus dynamique.

L'intérêt d'utiliser les exutoires karstiques est multiple. Ils se situent presque toujours en hauteur dans des massifs, ou en bordure de ceux-ci. Cette position facilitait l'acheminement de l'eau vers des sites qui, à l'époque romaine, étaient plutôt installés en position basse ; de ce fait, l'écoulement pouvait s'établir dans de bonnes conditions, avec des gradients altitudinaux contrôlables par le constructeur. Ces sources présentent également un régime souvent soutenu et régulier, ainsi qu'une bonne qualité des eaux, sauf en cas de "débourrages " consécutifs aux crues. Cela tient à la structure même des impluvia et des réservoirs karstiques où le rendement hydrique est, au niveau des exutoires, souvent optimum pour l'alimentation d'un aqueduc.

En Gaule méditerranéenne, les exemples de ce type sont nombreux et concernent tous les aqueducs de quelque importance ${ }^{10}$ en dehors du cas particulier de Glanum où un barragevoûte alimenté par un cours d'eau paraît avoir donné une altitude suffisante à la prise d'eau de l'aqueduc (AgustaBoularot, Paillet, 1997). Le dossier des prises et dérivations d'eau des rivières solidement argumentées est moins fourni et les hypothèses nombreuses, suite aux destructions naturelles et autres spoliations. Ce sont généralement des conduites de calibre modeste en milieu rural. M. Passelac évoque le ruisseau de la Rivière pour l'aqueduc de Bartissol, et la Font à Pierre pour celui de La Baronne ; le captage du ruisseau du Répudre est également envisagé pour l'aqueduc de Mailhac (Aude).

\section{L'APPORT DE L'ÉTUDE DES DÉPÔTS CARBONATÉS}

C'est l'aqueduc de Nîmes qui a suscité les premières recherches dans ce domaine, avant même son étude archéo-

8. Le cas est différent dans les pays de la Méditerranée au climat plus sec et aride, où les prises d'eau en rivières avec barrages-retenues en amont immédiat sont souvent la règle et dont on trouve des exemples en Espagne (aqueduc-rivière) : à Tolède-Guajaraz, Tarragone-Gaya, Ségovie-Frio, Sadaba-Arbas de Luesia, Pineda-Pineda. Toutefois cette thèse fait actuellement l'objet d'un débat (Feijoo Martinez, 2005) . I.es cas sont encore plus nombreux au Moyen-(Orient et au Maghreb où presque 200 prises d'eau en rivières ont été recensées en amont de barrages-retenues.

9. Hippocrate, Airs, eaux, lieux, VII-9, 10, 11.

10. Outre les exemples de ce dossier, on peut citer la source de (abezac pour l'aqueduc de Salleles-d Aude (Aude), celle de la Resclauze pour celui de Béziers (Hérault) (Ambert, (;illy el collab., 1989-1990; Espérou et al., 1994), la source des Sarrasins pour celui de l'oppidum de Mus à Sauve (Gard) (Blétry, Ferrand, 2002, p. 689-69(0), celle de Broussan pour celui de Bellegarde (Card) (Fabre, Saurage, 1999), la source du (iroseau pour le grand aqueduc de Vaison-la-Romaine (Vaucluse) (Dupuy, Chardon, 2003), celle de Rays pour l'aqueduc de Die (Drôme) (Grenier, 1960), la source de (hâteau-Bas pour le sanctuaire et l'agglomération antiques de Vernègues (Bouches-du-Rhône) (Fournier, Gazenbeck, 1999), les sources de Saint Antonin, de Vauvenargues, de la Trévaresse et de la Traconnade pour ceux d'Aix-en- 
logique (Gilly et al., 1971, 1978; Adolphe, 1973). Pour les spécialistes qui travaillent sur la formation des roches carbonatées, il s'agissait d'étudier les processus d'anthropisation du milieu sur un ouvrage ayant fonctionné durant une période qui apparaissait réduite à l'échelle chronologique des formations géologiques et qui donc permettait des observations plus précises. L'origine de cette collaboration spécifique aux ouvrages dont les eaux viennent d'un système karstique mérite d'être rappelée car elle n'est pas exportable en dehors des zones calcaires. Par la suite, la collaboration s'est précisée et affinée, à Cologne, Nîmes et Fréjus. Si, en effet, les dépôts carbonatés empêchent de lire correctement les architectures qu'ils ennoient à l'intérieur des conduits et recourrent d'un voile ou d'amas plus importants à l'extérieur, leur apport apparaît décisif au moins dans trois domaines :

- celui du fonctionnement de l'ouvrage, compte tenu de l'évolution des sections en long et en travers du cuvelage qui, en diminuant, affecte sérieusement le débit ;

- de la durée et des phases du fonctionnement, notamment par l'analyse microlithostratigraphique de l'accrétion des dépôts (voisine de $1 \mathrm{~mm} / \mathrm{an}$ ), de leur disposition transgressive et régressive (montée et baisse de l'eau) et des phases d'arrêts temporaires (discontinuités dans les faisceaux de lamines) ${ }^{11}$;

- et de la qualité biochimique des eaux écoulées (eaux claires = dépôts carbonatés clairs, bien lapidifiés ; eaux chargées de turbidités = dépôts carbonatés plus foncés, spongieux).

J.-L. Guendon, qui a été l'un des principaux acteurs de cette recherche, en a mis au point avec $\mathrm{J}$. Vaudour la méthodologie sur l'aqueduc de Nîmes. Il l'a affinée ultérieurement sur ceux d'Arles (Guendon, Villeminot, 1992 et Guendon, infra, p. 87-96) et de Fréjus (Gébara et al., 2002). Sa contribution au dossier, comme celle qui concerne l'aqueduc de Nîmes dans la Vistrenque, met en évidence l'intérêt d'une collaboration entre archéologues et naturalistes, et montre de manière concrète

Provence (Bouches-du-Rhône) (Franc, 1987), celles de la Foux et du Neisson pour celui de Fréjus (Var) (Gébara et al., 2002, p. 109-119), la source de Lombardi pour l'aqueduc nord-est de Cimiez à Nice (AlpesMaritimes) (Grenier, 1960).

Il en va de même pour les grands aqueducs antiques du pourtour de la Méditerranéc. Citons pour exemples : la source d'Alcabideque pour Conimbriga (Portugal) (Cunha, 1990), la source de Deifontes pour un aqueduc de Grenade, les sources d'Estella et du rio Verde pour ceux de Calahora et d'Almunecar (Espagne) (Casado, 1972), les sources de Zaghouan et Joukar pour (arthage (Tunisie) (Rakob, 1974), celle de Jadro pour Split (Croatie) (Nicod, 1996), de Karnarca pour VizeIstanbul (Cecem, 1996), d'Asku et du Madradag pour les aqueducs de Pergame (Garbrecht, 1979), de Suçikan Dere pour Antioche et d'Ikipinar Dere pour Xanthos (Turquie), les sources (Ein) Assafir, el Arrub, el Dilbeh, el Kuweiziba, el Daraj pour les aqueducs de Jérusalem, et Ein Shuni pour l'aqueduc supérieur de Caesarea Maritima (Israël) (Amit el al. dir., 2002) et en Italie, les sources de Montorio pour Vérone (Nicod, 1996), de Sassari (Manca, del Re) pour l'aqueduc sarde de Turris Libisonis (Satta, 2000), de la vallée de l'Aniene pour plus de la moitié des aqueducs de Rome.

11. Les lamines sont de fines strates carbonatées résultant d'une construction biochimique. I.es datations absolues uranium/thorium ou ${ }^{1+} \mathrm{C}$. réalisées sur des concrétions des aqueducs d'Arles (8 $\left.300 \mathrm{BP}\right)$ et de Fréjus (11 200 et $67000 \mathrm{BP})$ ne sont pour l'instant pas probantes et même totalement aberrantes ! l'apport de cette interdisciplinarité à la connaissance générale des aqueducs. Une approche similaire a renouvelé celle de l'hydraulique urbaine en Campanie romaine. En effet, Pompéi est alimenté par deux aqueducs, dits d'Avella et de Serino. On admettait que la construction du second aurait augmenté la quantité d'eau disponible pour cette ville. Mettant largement à contribution la géochimie des eaux, C. Ohlig (2001) en a réalisé une étude globale qui a montré qu'en réalité la quantité disponible avait diminué à la suite de la construction de l'aqueduc de Serino. Celui-ci n'est pas l'aqueduc d'une seule ville, Pompéi, mais un collecteur répartissant, entre Pompéi, Misène et les villes de Campanie, les eaux des sources de l'Apennin. Ohlig a ainsi pu réinterpréter le fonctionnement du castellum aquae et y distinguer deux états, dont le second assurait une meilleure répartition des quantités d'eau attribuées à Pompéi.

\section{PERSPECTIVES}

Ce dossier illustre la manière dont a été abordée l'étude des aqueducs dans des équipes archéologiques et par des archéologues qui, dans leur majorité, ne se sont pas spécialisés dans un type de monument mais l'envisagent dans le cadre d'études microrégionales sur l'occupation du sol. De ce fait, sans négliger les aspects techniques qui ont été abordés, nous évoquerons deux champs de la recherche qui nous semblent devoir être plus particulièrement parcourus: l'analyse diachronique des paysages et celle des usages de l'eau.

Les potentialités de l'étude d'un aqueduc pour une analyse diachronique des paysages sont liées à la nature même de cet ouvrage, monument linéaire qui s'inscrit dans des espaces variés pour lesquels il constitue un marqueur chronologique. Depuis une vingtaine d'années, des études de morphogenèse historique portant sur les basses vallées des cours d'eau et les piémonts ont mis à profit cette opportunité (Collectif, 1984). Inspirée par les mêmes principes, l'étude conduite par E. Fouache sur l'aqueduc de Nicopolis (Grèce) mériterait d'être généralisée (Doukellis et al., 1995). L'enfouissement d'ouvrages est révélateur d'une accrétion sédimentaire qui les " fossilise " par endroits ${ }^{12}$ : des épaisseurs non négligeables, atteignant 1 à $2 \mathrm{~m}$, sont régulièrement mises en évidence. Le recoupement des cours d'eau révèle des érosions de même ampleur: sur l'aqueduc de Nîmes (Pont-Roup et col de la Ratade), l'érosion différentielle régressive a déchaussé des piles porteuses sur des épaisseurs équivalentes, à la limite de petits bassins versants. De même, dans la montagne de la SainteVictoire et sur l'aqueduc d'Arles, des vestiges de tels ouvrages ont servi à évaluer les rythmes de l'érosion (Ballais, Crambes, 1993 ; Bellamy et al., 2000).

Dans un domaine proche, les aqueducs ont été utilisés comme des enregistreurs de néotectonique. Les recherches sur celui de Nîmes ont montré, en effet, que le rôle de la sismicité sur le devenir de l'ouvrage, qui n'avait pas été retenu dans un

12. Voir, par exemple, à Sallèles-d'Aude, sur l'aqueduc de Bellegarde (Gard), sur celui de Nîmes dans les vallées d'Eure, de l'Alzon et de la haute Vistrenque (Fabre, 2001 et observations de terrain inćdites). 
premier temps, devait, en fait, être pris en compte (voir infra, p. 73 ; Combes et al., 1997 ; Fabre, Levret, 2000), même si, a priori, la région est moins intéressante ${ }^{13}$ que d'autres sur le pourtour de la Méditerranée où néotectonique-sismotectonique et archéologie ont des connexions plus fortes ${ }^{14}$. Une analyse récente de l'aqueduc de Château-Bas à Vernègues plaide aussi fortement en ce sens ${ }^{15}$.

Cette collaboration interdisciplinaire s'impose aussi à l'échelle microrégionale pour tirer des conclusions sur les pratiques techniques que l'on cherche à reconstituer à partir de données sur des évolutions environnementales que l'on se propose d'établir. Ainsi, dans les Alpilles, les multiples observations qui ont porté sur les ouvrages hydrauliques témoignent de la longue durée de leur exploitation; toutes les investigations pratiquées dans les parties de l'aqueduc d'Arles situées en amont de Mas Créma n'ont livré que des canalisations modernes. Il convient donc de ne pas s'intéresser seulement aux ouvrages romains. La gestion des ressources en eau est évidemment une préoccupation bien antérieure à l'époque romaine. Dans une récente mise au point sur le peuplement du massif à l'époque protohistorique, P. Arcelin souligne la relation qui existe entre la carte des points d'habitat connus et celle " des potentialités hydrologiques, des résurgences et points d'eau bien plus nombreux au contact avec les piémonts et les plaines "(Gateau, Gazenbeek, 1999, p. 64).

Sur l'aqueduc de Nîmes, malgré de minutieuses recherches sur les nombreuses sources situées dans les environs de l'ouvrage, aucun cas de connexion au canal pour augmenter son débit n'a été repéré, alors que la plupart d'entre elles ont sans doute servi à l'alimentation d'établissements ruraux, signalés à proximité ${ }^{16}$ (Fabre et al. dir., 2000, p. 277). En fait,

13. De l'an Mil à nos jours, on dénombre "seulement " 11 cas d'intensité macrosismique à l'épicentre d'échelle MSK égale à VII (dégâts massifs à dommages notables) et 9 d'intensité supérieure à VII dans l'espace méditerranéen de la France (Lambert, Levret-Albaret, 1996); toutes intensités confondues, seuls la Drôme, le Vaucluse, et les Alpesde-Haute-Provence ont connu plus de 30 épisodes sismiques. Mais ces observations reflètent bien évidemment la carte de l'activité des chercheurs.

14. En particulier en Algérie, Espagne, Grèce et Turquic, où quelques études ont été conduites : Bousquet, Péchoux, 1977; Bousquet J.-C. et al., 1978 ; Collectif, 1983, 1984, p. 278-301 ; Bousquet B. et al., 1984; Jacques, 1984 ; Neboit dir., 1984 qui signalent des ruptures et des déformations par tectogenèse actuelle, cassante et souple, dans des structures hydrauliques et un aqueduc du sud de l'Espagne.

15. Recherches suite à une intervention de (;. Fabre en juillet 2004 (article à paraître dans $R A N, 38,2005$ ).

16. Deux exemples ont été récemment éclairés par la fouille, non loin du Pont du (Gard. Ainsi, une fouille préventive, pratiquéc aux abords de la source de Font-de-1)ringues (Vers-Pont-du-(rard), a révécé la présence c'est l'aqueduc lui-même qui, à certains moments, a été utilisé pour l'alimentation en eau des terres et des établissements voisins. Comme le montre l'article consacré ici à la Vistrenque, c'est une région où il est actuellement toujours en eau grâce à la présence d'une nappe superficielle abondante, et où, depuis plusieurs siècles, les regards ont servi à l'aménagement de nombreux puits pour l'irrigation. À l'occasion des prospections effectuées par M. Gazenbeek dans la vallée de l'Alzon, on a d'ailleurs observé que les servitudes liées à la présence de l'aqueduc n'avaient pas gêné l'occupation rurale: des bâtiments agricoles et même une petite villa ont été implantés à $10 \mathrm{~m}$ ou moins de l'ouvrage qui a pu, par ailleurs, favoriser l'installation de thermes (Benoit et al., 1994, p. 162).

Ces considérations débouchent sur une évidence méthodologique : la connaissance de ce type d'ouvrage de génie civil impose la confrontation des regards croisés de différents spécialistes : architecte, archéologue, géographe, géologue et géotechnicien, historien, hydraulicien, ingénieur, philologue, topographe... Il est d'ailleurs bien difficile d'en établir une liste exhaustive.

La propension que l'on a eue à attribuer aux Romains l'ensemble des réalisations dont ils ont été les initiateurs a conduit à dater de l'Antiquité des ouvrages plus récents. Ainsi, en Narbonnaise, l'aqueduc de Pézenas, un de ceux qu'étudie A. Grenier dans le volume où il traite des monuments des eaux, date en réalité de la fin du Moyen Âge ${ }^{17}$ (Soutou, 1986). En général, des fouilles et une étude complète permettent de lever les ambiguités. Mais, même dans le cas d'ouvrages dont l'Antiquité ne fait pas problème, de nombreuses imprécisions demeurent. Ces approches croisées que nous avons évoquées sont dans ce domaine d'un apport essentiel. En dehors de leurs contributions parfois décisives à la solution de ce problème, elles aident à comprendre qu'un aqueduc est un système complexe qui n'a pas nécessairement été conçu et réalisé d'un seul trait. Soumis par définition à l'action de l'eau, il a été l'objet de réfections et celles-ci ont pu avoir pour objectif de l'adapter à de nouveaux usages (Leveau, 2004).

d'un établissement viticole gallo-romain qui a produit des amphores (Provost et al., 1999, p. 733-734, n" 13*; Petitot et al., 2000). Par ailleurs, une fouille programmée sur la villa de la Gramière (Castillon-du-( ard) a été développée suite à la découverte d'une vaste citerne qui recucillait sans doute les eaux d'une source située au-dessus (Provost et al., 1999, p. 304-306, n*11*; Buffat, Guerre et collab., 2001, 2002).

17. D) autres exemples demeurent incertains: Vendres (Andrieu, 1999) et Saint-Thibéry dans l'Hérault (Lugand, Bermond, 2001), Ansignan daus les Pyrénéc-Orientales (Eydoux, 1979) Prade, 1988), Vinassan, Sigean et Fleury dans l'Aude (Dellong et collab., 2002), Saucetere et (avillargues dans le (aard (Prowost et al., 1999), Fontaine-de-Vaucluse dans le Vaucluse (Chardon, Mouraret, 1992), cte. 